\title{
Chemical basis underline the bioactivity fortification of licorice by honey-frying due to the natural deep eutectic solvent characteristics of honey
}

\section{Shasha Kong}

China Academy of Chinese Medical Sciences Institute of Chinese Materia Medica

\section{Pengyue Li}

China Academy of Chinese Medical Sciences Institute of Chinese Materia Medica

\section{Robert Verpoorte}

Leiden University Institute of Biology Leiden: Universiteit Leiden Instituut Biologie Leiden

\section{Jia Wang}

China Academy of Chinese Medical Sciences Institute of Chinese Materia Medica

\section{Chao Zhu}

Dezhou University

\section{Yuntao Dai ( $\nabla$ ytdai@icmm.ac.cn )}

China Academy of Chinese Medical Sciences Institute of Chinese Materia Medica

\section{Shilin Chen}

China Academy of Chinese Medical Sciences Institute of Chinese Materia Medica

\section{Research}

Keywords: Honey, Natural deep eutectic solvent (NADES), Licorice, Traditional Chinese medicine (TCM) processing, Bioavailability, Molecular interactions, Thermal stability

Posted Date: October 12th, 2021

DOI: https://doi.org/10.21203/rs.3.rs-915907/v1

License: (a) (1) This work is licensed under a Creative Commons Attribution 4.0 International License. Read Full License 


\section{Abstract \\ Background}

Honey has been widely used in medicine plants processing since ancient times. Honey-frying of herbal medicine is a well-known treatment in Chinese medicine. It has been reported that honey-frying can improve the immunomodulatory efficacy of licorice. However, it is still unknown why honey-frying improves the immunomodulatory activity of licorice. And our previous research demonstrated that honey has natural deep eutectic solvent (NADES) characteristics. NADES is a kind of solvent with broad polarity range and has attracted extensive attention of scholars in different fields. In the present study, we investigated chemical basis underline the possible potentiation of honey-frying on licorice to elucidate its chemical mechanism.

\section{Methods}

Firstly, immunological experiments were conducted to investigate whether the honey-processing could enhance the immunomodulatory efficacy of licorice. Then, the synergistic mechanism of honeyprocessed licorice was explored based on cell biological activity test, chemical composition test, bioavailability test, and Fourier transform-infrared (FT-IR) spectra.

\section{Results}

Pharmacological experiment verified that honey-processing enhanced the immunomodulatory efficacy of licorice. Moreover, honey increased the total flavonoid and polysaccharide contents in licorice decoction, improved the thermal stability and oral bioavailability of certain pharmacologically active constituents, and augmented their overall immunostimulatory functions. Similar effects of honey were also observed with NADES. The above effects are due to multiple molecular interactions between active compounds and sugar molecules of NADES.

\section{Conclusion}

These findings indicate that the biological activities of medicinal plants might be fortified by NADES/honey in the processing of the plant material. At the meantime, these findings provide theoretical and empirical basis for potential novel applications of honey or other NADESs at augmenting the healthpromoting effects of medicinal plants.

\section{Background}

Honey is a nutrient-dense food that is used globally. It is also used to process herbal medicines such as licorice and lily. Honey is well known from ancient times to modulate the function of herb preparations 
such as Lilii Bulbus (Lilium lancifolium Thunb.) and Licorice (Glycyrrhiza uralensis Fisch). Licorice, the root and rhizome of Glycyrrhiza uralensis Fisch., is widely used as TCM. It has been co-processed with honey since the Tang dynasty (A.D. 618-907) [1]. Since honey widely used in TCM processing, it is of key importance to understand the effect of honey-processing on the beneficial health effects of herbal medicines, which is vital for its innovative industrial applications. Modern pharmacological studies demonstrated that the immunomodulatory activity of licorice was improved after frying with honey [2,3]. However, it is still unknown why honey-frying improves the immunomodulatory activity of licorice, whether honey-frying affects the chemical composition or bioavailability of major beneficial phytochemicals in licorice.

Honey-fried licorice is prepared by soaking pieces of licorice roots in honey and frying the mixture [4]. The effects of honey-processing and heat-processing on the chemical constituents of licorice have been explored. The contents of liquiritin apioside (1) and liquiritin (2) in honey-fried licorice or fried licorice were lower than those in raw licorice [2, 5], while the contents of isoliquiritin apioside (4), isoliquiritin (5) and isoliquiritigenin (6) were relatively higher in honey-fried licorice, or in fried licorice than in raw licorice, concluding that isomerization reaction occurred during honey-frying [6]. It is also stated that during frying or honey-frying, the saponins and flavonoid glycosides in licorice decomposed to aglycones and sugars $[2,5,6]$. However, it is still unclear if honey affects the chemical composition of licorice during honeyfrying compared with fried licorice in the absence of honey.

Honey is a naturally occurring sweet viscous liquid that bees produce in the hive from the nectar they collect from flowers [7]. The main components of honey are various sugars and water [8-10]. Sugars account for $\sim 60-80 \%(w / w)$ and the water content of honey is usually $<20 \%(w / w)[11,12]$. The sugars and water in honey mainly contribute to its physicochemical properties. Honey was reported to act as a natural deep eutectic solvent (NADES) with a supramolecular structure [13-15]. Dai et al. [15-18] showed that NADES possess special solvent characteristics, which may improve the solubility, stability, and bioavailability of some natural products. Our previous work examined the role of honey in processing medicinal plant Astragalus propinquus Schischkin [18] and it showed that the honey-frying resulted in a change to a more favorable pharmacological activity. Hence, we speculate that honey-processed may change the chemical base of processed materials compared with frying without honey.

In the present study, we assessed whether honey-processing changed the immunomodulatory effects of licorice and investigated chemical basis underline the possible potentiation of honey-frying on licorice to elucidate its chemical mechanism. A targeted and a non-targeted approach were used for measuring the chemical profile of the decoctions of different processed licorice. To determine whether the NADES properties of honey modify bioactivities of licorice, a synthetic 35:35:1 (molar ratio) glucose:fructose:sucrose NADES was used as a control. The role of NADES in the dissolution, in-vivo absorption, and function of bioactive compounds in licorice were examined. The interaction between NADES and bioactive compounds from licorice was measured. The experiments performed herein confirmed that indeed the NADES itself affect the pharmacologic activity of the licorice. This study provided a scientific basis for the enhancement and potentiation of herbal medicine with NADES. 


\section{Materials And Methods \\ Sample preparation}

\section{Chemicals and reagents}

Raw licorice was purchased from Sichuan Xinhehua Decoction Pieces Co. Ltd. (Sichuan, China) and DNAAuthenticated as Glycyrrhiza uralensis Fisch root [19]. Voucher specimens (No. L20190706) were deposited in the herbarium of the Institute of Chinese Materia Medica of the China Academy of Chinese Medical Sciences. Honey was purchased from Beijing Tongrentang Bee Products Co. Ltd. (Zhejiang, China). Food-grade glucose, fructose, and sucrose were acquired from Sigma-Aldrich Corp. (St. Louis, MO, USA). Reference compounds including liquiritin (2), liquiritigenin (3), isoliquiritin (5), isoliquiritigenin (6), and glycyrrhizic acid (7) were obtained from Shanghai Yuanye Biotech Co. Ltd. (Shanghai, China). Isoliquiritin apioside (4), 2,4-dihydroxyacetophenone (9), D-fructose (10), and D-glucose (11) were obtained from Chengdu Chroma-Biotechnology Co. Ltd. (Chengdu, China). Liquiritin apioside (1), glycyrrhetinic acid (8), 5-hydroxymethylfurfural (12), and rutin, oleanolic acid (internal standard, IS) were procured from Beijing Century Aokang Biotech Co. Ltd. (Beijing, China). Their purity was determined by high-performance liquid chromatography (HPLC) to be $>98 \%$. Chromatography-grade acetonitrile and methanol were acquired from Fisher Scientific Worldwide Co. Ltd. (Shanghai, China). All other reagents were of analytical grade.

\section{NADES preparation}

The honey analog known as pure NADES was prepared by mixing a 35:35:1 molar ratio of glucose, fructose, and sucrose with $29 \mathrm{~mL}$ water [15]. The NADES product and honey were characterized by Bruker Ascend-600M ${ }^{1} \mathrm{H}$ Nuclear Magnetic Resonance (NMR) spectroscopy (Bruker Corp., karlsruhe, Germany), and by differential scanning calorimetry (DSC) [18].

\section{Preparation of different processed licorice and their decoctions}

Licorice processing. Processed licorice was prepared with dried licorice root, honey, and NADES. The licorice samples comprised raw licorice $(R)$ (unprocessed), fried licorice $(F)$ (stir-fried without additives), honey-fried licorice $(\mathrm{H})$ (mixed with honey and fried), and NADES-fried licorice $(\mathrm{N})$ (mixed with NADES and fried). The processing methods are described in the 2020 version of the "People's Republic of China Pharmacopoeia" (Provision No. 0213).

Licorice decoctions: Each of the foregoing products ( $100 \mathrm{~g}$ based on sliced licorice weight) was kept in $700 \mathrm{~mL}$ distilled water at $25^{\circ} \mathrm{C}$ for $30 \mathrm{~min}$ and refluxed at $100^{\circ} \mathrm{C}$ for $30 \mathrm{~min}$. The decoctions were filtered with 2 layers of gauze, and the residues were refluxed with $600 \mathrm{~mL}$ distilled water at $100^{\circ} \mathrm{C}$ for 20 min. The filtrates were combined, condensed to $<200 \mathrm{~mL}$ in $80{ }^{\circ} \mathrm{C}$ water bath, and adjusted to $200 \mathrm{~mL}$ with 
water. The decoctions were stored at $-80^{\circ} \mathrm{C}$ before use. The decoctions were resuspended by shaking before use in the cell-based bioactivity tests.

Reference solutions: Stock solutions of the reference standards (liquiritin apioside (1), liquiritin (2), liquiritigenin (3), isoliquiritin apioside (4), isoliquiritin (5), isoliquiritigenin (6), glycyrrhizic acid (7), glycyrrhetinic acid (8), 2,4-dihydroxyacetophenone (9), D-fructose (10), D-glucose (11), and 5hydroxymethylfurfural (12)) were prepared by dissolving $5 \mathrm{mg}$ of each standard in $5 \mathrm{~mL}$ methanol. The stock solutions were stored at $-20^{\circ} \mathrm{C}$ before use.

\section{Immunological experiment}

\section{Animals and experimental design}

Male ICR mice (18-22 g) were purchased from SPF Biotechnology Co. Ltd. (Beijing, China). They were housed in a specific pathogen-free grade laboratory at room temperature $\left(20-25^{\circ} \mathrm{C}\right)$ and constant relative humidity $(\mathrm{RH})$ under a 12-h light/12-h dark cycle. They had ad libitum access to food and distilled water. They were allowed to acclimate to their new environment for $3 \mathrm{~d}$ prior to the experiments. They were weighed and randomly divided into 10 groups ( $n=6$ per group), namely, normal, model, high-dose raw licorice $(7.5 \mathrm{~g} / \mathrm{kg})$, low-dose raw licorice $(5.0 \mathrm{~g} / \mathrm{kg})$, high-dose fried licorice $(7.5 \mathrm{~g} / \mathrm{kg})$, low-dose fried licorice $(5.0 \mathrm{~g} / \mathrm{kg})$, high-dose honey-processed licorice $(7.5 \mathrm{~g} / \mathrm{kg})$, low-dose honey-processed licorice (5.0 $\mathrm{g} / \mathrm{kg}$ ) groups, high-dose NADES-processed licorice $(7.5 \mathrm{~g} / \mathrm{kg})$, and low-dose NADES-processed licorice $(5.0 \mathrm{~g} / \mathrm{kg})$ groups. Mice in group 2-10 were intragastrically (i.g.) administrated with rhubarb extract (15 $\mathrm{g} / \mathrm{kg}$, once a day) for $7 \mathrm{~d}$ to induce Pi-deficiency model. Then, the mice in group 1 (normal) and group 2 (model) were administered (i.g.) sterile physiological saline once daily for $7 \mathrm{~d}$. Mice in the raw, fried, honey-processed, and NADES-processed licorice groups were orally administered decoctions at 7.5 $\mathrm{mL} / \mathrm{kg}$ body weight (BW) once daily for $7 \mathrm{~d}$. All procedures were conducted in strict accordance with the Guide for the Care and Use of Laboratory Animals of the Ministry of Science and Technology of China (2006) and approved by the Animal Welfare Ethics Committee of the Institute of Chinese Materia Medica (China Academy of Chinese Medical Sciences, Beijing, China).

\section{Sample collection and measurement}

Twenty-four hours after the final administration, the mice were weighted and then injected in the cauda with India ink $(0.01 \mathrm{~mL} / \mathrm{g} \mathrm{BW})$. Twenty microliters blood were taken from the eyepit vein plexus at 2 min and $20 \mathrm{~min}$ after the India ink injection. The blood was placed in a centrifuge tube containing $2 \mathrm{~mL}$ of $1 \%$ $(\mathrm{w} / \mathrm{v})$ sodium bicarbonate for $1 \mathrm{~h}$. The absorbances (optical density [OD]) of the mice sera were measured at $650 \mathrm{~nm}$ by Thermo Scientific Multiskan GO Multiskan Spectrum (Thermo Fisher Scientific, Waltham, MA, USA). Thirty minutes after the India ink injections, Mice were euthanized, then the spleen and thymus were excised and weighed, 


\section{Data analysis}

The charcoal particle expurgation index $(\mathrm{K})$ of the macrophage count was calculated as follows:

$$
\mathrm{K}=(\log \mathrm{OD} 1-\log \mathrm{OD} 2) /(\mathrm{t} 2-\mathrm{t} 1)
$$

where, $t_{1}$ and $t_{2}$ are the time points ( 2 min and 20 min, respectively) at which blood was drawn from the mouse eyes after the India ink injection.

The spleen index was calculated as follows [2]:

Spleen index $(\mathrm{mg} / \mathrm{g})=$ spleen weight $(\mathrm{mg}) /$ body weight $(\mathrm{g})$

Data were expressed as means \pm SD. Differences between groups were evaluated with SPSS v. 25.0 (SPSS Inc., Chicago, IL, USA). $P<0.05$ was considered statistically significant.

\section{Cell-based bioactivity tests}

\section{Cell culture}

HEK-293 cells were grown in Dulbecco's modified Eagle's medium (DMEM) (Life Technologies, Carlsbad, CA, USA) containing $10 \%(\mathrm{v} / \mathrm{v}$ ) fetal bovine serum (FBS) and $100 \mu \mathrm{g} / \mathrm{mL}$ kanamycin in an incubator at 37 ${ }^{\circ} \mathrm{C}$ under a humidified $\mathrm{CO}_{2}(5 \%)$ atmosphere. Before seeding, pER-a-Luc HEK-293 cells were washed with phosphate-buffered saline (PBS) and cultured in DMEM containing $5 \%$ cdFBS for $2 \mathrm{~d}$ starvation. The pERa-Luc HEK-293 cells were seeded in a 96-well plate. Each well contained 40,000 cells and $0.1 \mathrm{~mL}$ estrogen-free medium. Other cell lines including pNrf2-Luc HEK-293 and pTLR-4-Luc HEK-293 were inoculated in 96-well plates. Each well contained 20,000 cells and $0.1 \mathrm{~mL}$ DMEM with $10 \%$ (v/v) FBS.

\section{Biological activity testing}

The estrogen receptor (ER)- $a$, nuclear factor erythroid 2-related factor (Nrf2), and toll-like receptor 4 (TLR4) promoter assays were conducted on the HEK293 cells. An appropriate licorice extract concentration was added to the pER- $\alpha$-Luc HEK-293 cells, and the suspension in a humidified $\mathrm{CO}_{2}(5 \%)$ incubator at $37^{\circ} \mathrm{C}$ was incubated for $24 \mathrm{~h}$. Then, $17-\beta$-estradiol (Sigma-Aldrich Corp.) was dissolved in dimethyl sulfoxide (DMSO) and used as a positive control. The medium was aspirated before adding 0.5 $\mathrm{mL}$ lysis buffer ( $\mathrm{pH} \mathrm{7.8)}$ to each well for $10 \mathrm{~min}$. Luciferase assay buffer $(0.1 \mathrm{~mL} /$ well) was added, and the luciferase activity was immediately determined. Estrogenic activity was calculated as follows [20]: 
Estrogenic activity $(\%)=[($ sample absorbance at $560 \mathrm{~nm}-$ control absorbance at $560 \mathrm{~nm}) /(17-\beta-$

estradiol absorbance at $560 \mathrm{~nm}-$ control absorbance at $560 \mathrm{~nm}$ )] $\times 100 \%$

For the TLR4 promoter assay, an appropriate licorice extract concentration was added to the pTLR4-Luc HEK-293 cells, and they were incubated for $4 \mathrm{~h}$ at $37^{\circ} \mathrm{C}$. Lipopolysaccharide (LPS) (Sigma-Aldrich Corp.) dissolved in DMSO was the positive control. For the Nrf2 promoter assay, an appropriate licorice extract concentration was added to the pNrf2-Luc HEK 293 cells, and they were incubated for $16 \mathrm{~h}$ at $37^{\circ} \mathrm{C}$. Andrographolide $(20 \mu \mathrm{M})$ dissolved in DMSO was the positive control. The other steps followed were the same as those used for the ER- $a$ assay.

\section{Total polysaccharide and flavonoid content measurement}

The levels of total polysaccharides and flavonoids in the decoctions were determined by UV-Vis spectrophotometry [21]. The total polysaccharides were detected at $590 \mathrm{~nm}$ by the anthrone-sulfuric acid method using anhydrous glucose as a reference [22]. Liquiritin was the reference, as it is the main dihydroflavone in licorice. All major flavonoids in licorice have maximum absorptions at $336 \mathrm{~nm}$.

\section{Metabolomics analysis}

\section{Non-target metabolomics analysis with UHPLC-Q-Orbitrap MS}

The licorice decoctions were thawed, diluted 20,000-fold with methanol, and filtered through a $0.22 \mu \mathrm{m}$ membrane before analysis on an Ultimate 3000 ultrahigh-performance liquid chromatography (UHPLC) instrument coupled with a Thermo Q-ExActive ${ }^{T M}$ Plus Orbitrap ${ }^{T M}$ high-resolution mass spectrometer (Thermo Fisher Scientific) fitted with a heat electrospray ionization (HESI) interface. Samples were separated on an Acquity UPLCHSS T3 C18 column $(2.1 \mathrm{~mm} \times 100 \mathrm{~mm} ; 1.8 \mu \mathrm{m}$; Waters Corp., Milford, CT, USA). The column temperature was maintained at $40{ }^{\circ} \mathrm{C}$. The flow rate was $0.30 \mathrm{~mL} / \mathrm{min}$. Mobile phase $\mathrm{A}$ was $0.1 \%(\mathrm{v} / \mathrm{v})$ formic acid-acetonitrile, and mobile phase $B$ was $0.1 \%(\mathrm{v} / \mathrm{v})$ formic acid. The gradient program was as follows: 0-1 min, 98\% B; 1-2.5 min, 98-95\% B; 2.5-4 min, 95-70\% B; 4-8 min, 70-65\% B; 8-15 min, 65-50\% B; 15-18 min, 50-0\% B; 18-23 min, 0\% B; 23-23.5 min, 0-98\% B; 23.5-26 min, $98 \% \mathrm{~B}$. The sample temperature was maintained at $20^{\circ} \mathrm{C}$, and the injection volume was $5 \mu \mathrm{L}$.

The operating conditions of the mass spectrometry (MS) were as follows: HESI source in positive and negative mode; capillary temperature, $320^{\circ} \mathrm{C}$; sheath gas flow, $35 \mathrm{arb}$; auxiliary gas flow, $10 \mathrm{arb}$; scan modes, full MS and dd-MS²; full MS, 70,000 resolution; full MS residence time, 150 ms; dd-MS², 17,500 resolution; dd-MS ${ }^{2}$ TopN, 5; mass spectra recorded in $\mathrm{m} / \mathrm{z}$ range, 120-1,000; step collision energy, 20 $\mathrm{V} / 45 \mathrm{~V} / 70 \mathrm{~V}$; positive mode, $3.5 \mathrm{kV}$ spray voltage; negative mode, $3.2 \mathrm{kV}$ spray voltage. Data were analyzed with Compound Discoverer v. 3.0 (Thermo Fisher Scientific). 
The raw mass data were pre-processed with Progenesis QI v. 1.0 (Waters Corp., Milford, CT, USA) for peak alignment and selection as well as deconvolution. Individual ion fragment intensities were normalized for all compounds. The data MS matrices were imported into SIMCA-P v. 13 (Umetrics, Umeå, Sweden) for principal component analysis (PCA) by Pareto scaling to identify group distributions, perform orthogonal partial least square discriminant analysis (OPLS-DA), and identify the potential quality difference markers among groups by variable importance in projection (VIP). One-way ANOVA was conducted on the ion response strength data of the potential mass difference markers using GraphPad Prism v. 7.0 (GraphPad Software, La Jolla, CA, USA) to identify any significant differences among potential mass difference markers. The screened quality difference markers were identified by chromatographic comparison against reference substances, laboratory databases, and literature reports $[6,23,24]$.

\section{Quantitative analysis with UHPLC-QqQ-MS}

Licorice decoctions were thawed, diluted 20,000-fold with methanol, and filtered through a $0.22 \mu \mathrm{m}$ membrane before quantitative analysis. The assay was performed on a 6500 Plus Triple Quad LCMS/MS system fitted with an ExionLC UHPLC unit (AB SCIEX Corp., Framingham, MA, USA). Sample components were separated on an Acquity UPLCHSS T3 C18 column ( $2.1 \mathrm{~mm} \times 100 \mathrm{~mm}$; $1.8 \mu \mathrm{m}$; Waters Corp.). The column temperature was maintained at $40{ }^{\circ} \mathrm{C}$, and the flow rate was $0.30 \mathrm{~mL} / \mathrm{min}$. Mobile phase $A$ was $0.1 \%(\mathrm{v} / \mathrm{v})$ formic acid-acetonitrile. Mobile phase $B$ was $0.1 \%(\mathrm{v} / \mathrm{v})$ formic acid. The gradient program was as follows: 0-3 min, 98-95\% B; 3-4 min, 95-81\% B; 4-7 min, 81-70\% B; 7-9 min, 70\% B; 9-10 min, 70-50\% B; 10-12 min, 50-45\% B; 12-17 min, 45-5\% B; 17-22 min, 5\% B; 22-23 min, 5-98\% $\mathrm{B} ; 23-27 \mathrm{~min}, 98 \% \mathrm{~B}$. The sample temperature was maintained at $20^{\circ} \mathrm{C}$. The injection volume was $5 \mu \mathrm{L}$.

MS data were recorded using electrospray ionization (ESI), negative ion detection, and multiple reaction monitoring (MRM) scanning. The ion spray voltage and temperature were set at $4,500 \mathrm{~V}$ and $500{ }^{\circ} \mathrm{C}$, respectively. The curtain, gas 1 (nebulizer), and gas 2 (heater) gas pressures were set to $35 \mathrm{psi}, 50 \mathrm{psi}$, and $50 \mathrm{psi}$, respectively. The collision gas pressure was set to $9 \mathrm{psi}$. The compound dwell time was set to $10 \mathrm{~ms}$. The entrance potential and collision cell exit potential were set to $10 \mathrm{~V}$ and $18 \mathrm{~V}$, respectively. Data were acquired with Analyst v. 1.6.3 (AB SCIEX Corp., Framingham, MA, USA). The MS/MS parameters for the 18 compounds are listed in Table S1. The method was validated (Supplementary Material).

\section{Pharmacokinetic experiments}

\section{Drug administration and sample preparation}

Male Sprague-Dawley (SD) rats (220-250 g) were obtained from HFK Bioscience Co. Ltd. (Beijing, China). The rats were bred at $25^{\circ} \mathrm{C}, 60 \pm 5 \% \mathrm{RH}$, and 12-h dark-light cycle for $3 \mathrm{~d}$. They had ad libitum access to water and normal chow. All animals were fasted overnight before the experiments. The rats were randomly divided into four groups ( $n=6$ per group). The raw licorice decoction (R; $1.0 \mathrm{~g} / \mathrm{mL}$ raw licorice 
equivalent), the fried licorice decoction ( $F ; 1.0 \mathrm{~g} / \mathrm{mL}$ raw licorice equivalent), the honey-fried licorice decoction $(\mathrm{H} ; 1.0 \mathrm{~g} / \mathrm{mL}$ raw licorice equivalent), and the NADES-processed licorice decoction $(\mathrm{N} ; 1.0 \mathrm{~g} / \mathrm{mL}$ raw licorice equivalent) were orally administered to the rats at $8 \mathrm{~g} / \mathrm{kg}$. Blood samples $0.3 \mathrm{~mL}$ in volume were collected from the angular vein into heparinized tubes at $0.08 \mathrm{~h}, 0.25 \mathrm{~h}, 0.5 \mathrm{~h}, 1 \mathrm{~h}, 2 \mathrm{~h}, 4 \mathrm{~h}, 6 \mathrm{~h}, 8 \mathrm{~h}$, $12 \mathrm{~h}$, and $24 \mathrm{~h}$ after oral decoction administration. They were immediately centrifuged at $12,000 \mathrm{rpm}$ at $4{ }^{\circ} \mathrm{C}$ for $3 \mathrm{~min}$, and the plasma was stored at $-80^{\circ} \mathrm{C}$ until use.

Calibration standard (CS) and quality control (QC) samples were prepared (Supplementary Materials). One hundred microliters of each plasma, CS, or QC sample was spiked with $300 \mu \mathrm{L}$ acetonitrile (including rutin and oleanolic acid; $100 \mathrm{ng} / \mathrm{mL}$ ). The mixture was vortexed for 3 min and centrifuged at 12,000 rpm and $4{ }^{\circ} \mathrm{C}$ for $10 \mathrm{~min}$. The supernatant was collected and evaporated under a nitrogen stream in a $37^{\circ} \mathrm{C}$ water bath. The residue was redissolved in $100 \mu \mathrm{L}$ acetonitrile, vortexed for $3 \mathrm{~min}$, and centrifuged at $12,000 \mathrm{rpm}$ at $4{ }^{\circ} \mathrm{C}$ for $10 \mathrm{~min}$.

\section{UHPLC-QqQ-MS analysis}

The assay was performed on a 6500 Plus Triple Quad LC-MS/MS system fitted with an ExionLC UHPLC unit (AB SCIEX Corp., Framingham, MA, USA). Sample components were separated on an Acquity UPLCHSS T3 C18 column ( $2.1 \mathrm{~mm} \times 100 \mathrm{~mm} ; 1.8 \mu \mathrm{m}$; Waters Corp.). The column temperature was maintained at $40{ }^{\circ} \mathrm{C}$, and the flow rate was $0.30 \mathrm{~mL} / \mathrm{min}$. Mobile phase $A$ was $0.1 \%(\mathrm{v} / \mathrm{v})$ formic acidacetonitrile. Mobile phase $B$ was $0.1 \%(\mathrm{v} / \mathrm{v})$ formic acid. The gradient program was as follows: $0-3 \mathrm{~min}$, 98-95\% B; 3-4 min, 95-81\% B; 4-7 min, 81-70\% B; 7-9 min, 70\% B; 9-10 min, 70-50\% B; 10-12 min, $50-45 \%$ B; $12-17$ min, 45-5\% B; 17-22 min, 5\% B; 22-23 min, 5-98\% B; 23-27 min, 98\% B. The sample temperature was maintained at $20^{\circ} \mathrm{C}$. The injection volume was $5 \mu \mathrm{L}$. The electrospray ionization (ESI) source was set in negative ion detection. Quantitation was performed in multiple reaction monitoring (MRM) mode. The transition ions were $m / z 549.1 \rightarrow 255.2$ for liquiritin apioside (1), $m / z 417.1 \rightarrow 134.9$ for liquiritin (2), $m / z 255.1 \rightarrow 119.1$ for liquiritigenin (3), $m / z \quad 469.3 \rightarrow 425.4$ for glycyrrhetinic acid (8), and $m / z$ $609.2 \rightarrow 300.2$ for rutin (IS), $m / z 455.4 \rightarrow 407.5$ for oleanolic acid (IS). The collision energy (CE) for each compound and IS were $-44 \mathrm{~V},-43 \mathrm{~V},-32 \mathrm{~V},-53 \mathrm{~V}$, and $-52 \mathrm{~V},-57 \mathrm{~V}$, respectively. The declustering potential (DP) were set at $-80 \mathrm{~V}$. The ion spray voltage and temperature were set at $4,500 \mathrm{~V}$ and $500{ }^{\circ} \mathrm{C}$, respectively. The curtain, gas 1 (nebulizer), and gas 2 (heater) gas pressures were set to $35 \mathrm{psi}, 50 \mathrm{psi}$, and $50 \mathrm{psi}$, respectively. The collision gas pressure was set to 9 psi. The compound dwell time was set to $10 \mathrm{~ms}$. The entrance potential and collision cell exit potential were set to $10 \mathrm{~V}$ and $18 \mathrm{~V}$, respectively. Data were acquired with Analyst v. 1.6.3 (AB SCIEX Corp., Framingham, MA, USA). The method was validated (Supplementary Material).

\section{Statistical analysis}

The pharmacokinetic parameters were calculated with Drug Analysis System v. 3.2.6 (Mathematical Pharmacology Professional Committee of China). Noncompartmental analysis was used to determine 
the maximum time $\left(T_{\text {max }}\right)$, maximum plasma concentration $\left(C_{\text {max }}\right)$, and area under the curve $\left(\mathrm{AUC}_{0-}\right.$ $12 / 24 \mathrm{~h})$. Data were means \pm SD. Statistical analyses of all data were performed with one-way ANOVA (SPSS v. 25.0; SPSS Corp., Chicago, IL, USA).

\section{Molecular interaction determination of honey and its mixture with licorice compounds}

\section{Sample preparation}

Honey and corresponding NADES were obtained as described in Subsection of sample preparation. A fructose solution was obtained by dissolving $2 \mathrm{~g}$ fructose in $0.5 \mathrm{~mL}$ water to ensure that the final water content was the same as that in NADES. Glucose hydrate was prepared in the same manner as the fructose solution. The mixture of liquiritin and NADES was obtained by dissolving $0.12 \mathrm{~g}$ liquiritin in $1 \mathrm{~mL}$ NADES and stirring thoroughly.

\section{Apparatus and analysis}

Fourier transform-infrared (FT-IR) spectra (range 4,000-600 $\mathrm{cm}^{-1}$ ) were recorded at $25^{\circ} \mathrm{C}$ in a Frontier FTIR spectrometer (PerkinElmer, Waltham, MA, USA). The spectra were baseline-corrected with Specture v. 10 (PerkinElmer).

\section{Results}

\section{Effects of licorice on mouse immune function}

The effects of aqueous decoctions of raw licorice $(R)$, fried licorice $(F)$, honey-fried licorice $(H)$, and NADES-fried licorice $(\mathrm{N})$ on murine immunity were evaluated using a carbon clearance test according to the reference [2] with some modification. Compared with the normal group, mice in other groups showed obvious behavioral abnormalities after 5 days of intragastric administration of rhubarb decoction, including weight loss, dry and dull hair, listless expression and thin stool, which indicates that the Pideficiency has been successfully built. Decoctions of honey-fried licorice and NADES-fried licorice both increased the phagocytic, spleen and thymus indices of Pi-deficiency mice, while in the F group, only the high dosage group was effective in improving the expurgation indices of Pi-deficiency mice.

There were no differences between honey-fried licorice and NADES-fried licorice. Compared with the highdose raw licorice group, the expurgation and spleen indices of $\mathrm{H}$ and $\mathrm{N}$ groups at the same dosage were significantly higher $(P<0.05)$ (Table 1$)$, indicating that the immune enhancement effect of honey-fried and NADES-fried licorice are better than fried licorice. Honey alone had no immunostimulant effect in 
mice $[2,3]$. These findings indicate that honey-processing and NADES- processing could enhance the licorice immunomodulation action.

Table 1

The expurgation index, spleen index and thymus index of mice after oral administration of physiological saline, licorice decoction, fried licorice decoction, honey- fried licorice decoction and NADES-fried licorice decoction for 7 days. (mean $\pm S D, n=6$ )

\begin{tabular}{|c|c|c|c|c|}
\hline Group & $\begin{array}{l}\text { Dose } \\
(\mathrm{g} / \mathrm{kg})\end{array}$ & $\begin{array}{l}\text { Expurgation index } \\
\left(\times 10^{-2}\right)\end{array}$ & $\begin{array}{l}\text { Spleen index } \\
(\mathrm{mg} / \mathrm{g})\end{array}$ & $\begin{array}{l}\text { Thymus index } \\
(\mathrm{mg} / \mathrm{g})\end{array}$ \\
\hline Model & I & $2.73 \pm 0.57$ & $3.07 \pm 0.31$ & $1.96 \pm 0.26$ \\
\hline Normal & I & $3.11 \pm 0.44$ & $3.17 \pm 0.34$ & $2.05 \pm 0.33$ \\
\hline \multirow[t]{2}{*}{ Raw licorice (R) } & 5 & $3.09 \pm 0.51$ & $3.26 \pm 0.39$ & $2.09 \pm 0.39$ \\
\hline & 7.5 & $3.02 \pm 0.42$ & $3.24 \pm 0.25$ & $2.16 \pm 0.26$ \\
\hline \multirow[t]{2}{*}{ Fried licorice $(F)$} & 5 & $3.13 \pm 0.54$ & $3.35 \pm 0.35$ & $2.07 \pm 0.36$ \\
\hline & 7.5 & $3.32 \pm 0.29^{*}$ & $3.39 \pm 0.38$ & $2.17 \pm 0.37$ \\
\hline \multirow{2}{*}{$\begin{array}{l}\text { Honey-fried licorice } \\
\text { (H) }\end{array}$} & 5 & $3.50 \pm 0.46^{*}$ & $3.58 \pm 0.36^{*}$ & $2.16 \pm 0.44$ \\
\hline & 7.5 & $3.73 \pm 0.49^{\star \#}$ & $3.72 \pm 0.29^{\star \#}$ & $2.39 \pm 0.42^{*}$ \\
\hline \multirow{2}{*}{$\begin{array}{l}\text { NADES-fried licorice } \\
(\mathrm{N})\end{array}$} & 5 & $3.52 \pm 0.47^{\star}$ & $3.58 \pm 0.39^{*}$ & $2.13 \pm 0.34$ \\
\hline & 7.5 & $3.81 \pm 0.41^{\star \#}$ & $3.77 \pm 0.43^{\star \#}$ & $2.34 \pm 0.28^{*}$ \\
\hline
\end{tabular}

\section{Honey-frying improved licorice bioactivity in certain signal transduction pathways}

To investigate the effects of honey on licorice immunomodulation, the biological effects of $R, F, H$, and $N$ on the endocrine-related ER- $a$, TLR4, and Nrf2 signaling pathways were compared. The bioactivities increased with $\mathrm{H}$ and $\mathrm{N}$ compared with those of the R. There were no differences between $\mathrm{H}$ and $\mathrm{N}$. However, both showed significantly higher activity than F in ER-a and Nrf2 signaling pathways (Fig. 1). Hence, NADES-frying and honey-frying substantially improved licorice bioactivity by a mechanism common to both treatments.

\section{Effect of honey-frying on the dissolution of total flavonoids and polysaccharides}

Figure 2a shows that there was higher total flavonoid content in $\mathrm{H}$ and $\mathrm{N}$ than in $\mathrm{R}$. In contrast, $\mathrm{F}$ had relatively lower total flavonoid content. There was higher total polysaccharides content in $\mathrm{F}, \mathrm{H}$, and $\mathrm{N}$ than 
in $\mathrm{R}$. There were higher levels of total polysaccharides in $\mathrm{H}$ and $\mathrm{N}$ than in $\mathrm{F}$. Therefore, frying without honey or NADES decreased total flavonoid content and increased total polysaccharides content in licorice. Honey and NADES avoided the total flavonoid loss caused by frying, and even increased the level of total flavonoid compared with R. $\mathrm{H}$ and $\mathrm{N}$ did not significantly differ in terms of total polysaccharide or flavonoid content.

\section{Effects of honey-frying on the chemical composition of licorice decoctions}

\section{Different chemical profiles observed by non-targeted metabolomics}

The licorice decoctions were analyzed by UHPLC-QE-Orbitrap-MS. PCA of the MS data showed three distinct groups, representing R, F and $\mathrm{N}$ plus $\mathrm{H}$ as one group (Fig. 2b). Processing altered the chemical composition of the licorice. Moreover, the chemical composition of $\mathrm{H}$ was significantly different from that of $\mathrm{F}$ but resembled that of $\mathrm{N}$. Thus, heating and honey significantly affected the chemical profile of the licorice decoction, while honey-frying and NADES-frying had similar effects.

\section{Quantitative analysis by LC-QqQ-MS}

Eighteen potential quality markers were identified by analyzing the UHPLC-Q-Orbitrap MS data by OPLSDA (Table S1). Then, LC-QqQ-MS was used to perform targeted quantitative analyses on twelve markers with reference substances. It was also applied towards pseudo-target quantitative analysis of six markers without reference substances.

The flavonoid levels significantly differed among the licorice decoctions from the R, F, N, and $\mathrm{H}$ groups. Fried samples $\mathrm{F}$ had relatively lower levels of dihydroflavone (liquiritin apioside (1), liquiritin (2),liquiritigenin (3), 6"-O -acetylliquiritin apioside (13), 6"-O-acetylliquiritin (15)) and aglycone isoliquiritigenin (6) than control R. Whereas higher levels of chalcone (isoliquiritin apioside (4), isoliquiritin (5), 6"-O-acetylisoliquiritin apioside (14), and 6"-O-acetylisoliquiritin (16)) and 2,4dihydroxyacetophenone (9) were observed in control $\mathrm{F}$ than control R. Moreover, compared with control R there were higher degrees of increase in chalcones $(4,14,16)$ and 2,4-dihydroxyacetophenone $(9)$ in $F$ than in $\mathrm{H}$ or $\mathrm{N}$ (Fig. 3a and S1).

The levels of the saponins glycyrrhizic acid (7), uralsaponin B (17), and 22 $\beta$-acetoxylglycyrrhizic acid (18) decreased in F, H, N (Fig. 3a and S1). However, the glycyrrhetinic acid (8) content was significantly increased after processing $(F, H, N)$. Compared to control $R$, there was a greater decrease in the glycyrrhizic acid (7) content in $\mathrm{F}$ than in $\mathrm{H}$ or $\mathrm{N}$ (Fig. 3a). Obviously, the reducing sugars $D$-fructose (10), and D-glucose (11) were the major compounds in the honey and NADES processed samples, 5hydroxymethylfurfural (12) was formed in connection with the heating.

\section{Effects of honey-frying on the absorption of representative chemical components in licorice}


A detection method for plasma liquiritin apioside (1), liquiritin (2), liquiritigenin (3), and glycyrrhetinic acid (8) was established according to the reference [25] with some modification and validated. Table S5 and S6 demonstrate that the method was reproducible and accurate and could be applied for measuring these four compounds in rat plasma.

The plasma liquiritin apioside (1), liquiritin (2), liquiritigenin (3), and glycyrrhetinic acid (8) concentrations in SD rats were monitored within $24 \mathrm{~h}$ after the administration of a single oral dose of licorice. Liquiritin apioside (1), liquiritin (2), liquiritigenin (3), could not be detected after 24 hours of oral administration. Considering the measurement results of the levels of the major compounds in the plasma of mice (Fig. 4 and Table S7), it seems that for the major compounds measured (1, 2, 3, and $\mathbf{8})$, the flavonoids $\mathbf{1}$ and $\mathbf{3}$ showed higher plasma levels in the presence of the honey or the NADES (Fig. 4), the same applies for glycyrrhetinic acid (8). For the $F$ samples no increase of the plasma level was observed, in fact the curve is identical with the control (Fig. 4). The $\mathrm{H}$ and $\mathrm{N}$ samples show an increased plasma level of compounds 1, 3, 8 if compared with both the control $R$ and the $F$ samples, which indicates that the increased plasma levels of compounds is correlated with the presence of the sugars in $\mathrm{H}$ and $\mathrm{N}$.

The $T_{\max }$ of liquiritin apioside (1) in $\mathrm{H}$ and $\mathrm{N}$ group was later than that in $\mathrm{R}$ and $\mathrm{F}$ group, and the $\mathrm{AUC}_{0}$ 12h of liquiritin apioside (1) significantly increased in $\mathrm{H}$ and $\mathrm{N}$ group. For liquiritin (2), and liquiritigenin (3), there was a double absorption peak, and the $C_{\max }$ significantly increased in the honey-and NADES-fried licorice decoctions as well as the increased $\mathrm{AUC}_{0-12 \mathrm{~h}}$ of liquiritigenin (3). The $C_{\max }$ and $\mathrm{AUC}_{0-24 \mathrm{~h}}$ of glycyrrhetinic acid (8) were higher for the honey and NADES.

\section{Mechanism analysis of the effects of honey-frying on licorice}

FT-IR spectra were recorded for honey, NADES, glucose, and fructose to elucidate the structure similarity of NADES and honey. Absorption bands were similar for honey and NADES in terms of peak strength, position, and shape (Fig. 5a). Hence, honey and NADES have similar physico-chemical compositions [18]. Compared with the peaks of FT-IR spectra for the ingredients of NADES (glucose and fructose), wide and shifted peaks were observed in NADES spectra, suggesting that glucose and fructose have molecular interactions in the NADES. The stretching vibration absorption bands of the $\mathrm{C}-\mathrm{O}\left(v_{\mathrm{C}-\mathrm{O}}\right)$ in glucose and fructose were strong, sharp peaks at $1,008 \mathrm{~cm}^{-1}$ and $1,047 \mathrm{~cm}^{-1}$, respectively, whereas in that of NADES a broad peak at $1,028 \mathrm{~cm}^{-1}$ was observed. The deformation vibration absorption bands of the $\mathrm{O}-\mathrm{H}\left(\delta_{\mathrm{O}-\mathrm{H}}\right)$ in glucose and fructose shifted from $915 \mathrm{~cm}^{-1}$ to $897 \mathrm{~cm}^{-1}$ and from $923 \mathrm{~cm}^{-1}$ to $919 \mathrm{~cm}^{-1}$, respectively, in NADES with broaden peak shape. These results show that the hydroxyl groups in the glucose and fructose of NADES have inter-molecular interactions including hydrogen bonds. The sharp peaks of the deformation vibration absorption bands of the $\mathrm{C}-\mathrm{H}\left(\delta_{\mathrm{C}-\mathrm{H}}\right)$ in glucose shifted from $1,380 \mathrm{~cm}^{-}$ 1 to a broad peak at $1,344 \mathrm{~cm}^{-1}$ in NADES. In NADES, several sharp peaks of the stretching vibration absorption bands of $\mathrm{C}-\mathrm{H}\left(v_{\mathrm{C}-\mathrm{H}}\right)$ in glucose and fructose between $2,900 \mathrm{~cm}^{-1}$ and $3,000 \mathrm{~cm}^{-1}$ were 
replaced by a broad band around $2,928 \mathrm{~cm}^{-1}$. These spectral characteristics of NADES reveal its supramolecular structure with multiple interactions between the glucose and fructose molecules.

FT-IR spectra were recorded for NADES, liquiritin, and a mixture of liquiritin and NADES to clarify the mechanism by which NADES affects the solutes. The spectra (Fig. 5b) showed various absorption bands for the functional groups in liquiritin, including the stretching vibration band of $C=O\left(v_{C=0}\right)$ at $1,604 \mathrm{~cm}^{-1}$, the stretching vibration band of $\mathrm{C}-\mathrm{O}-\mathrm{C}\left(v_{\mathrm{C}-\mathrm{O}-\mathrm{C}}\right)$ at $1,229 \mathrm{~cm}^{-1}$, and the deformation vibration band of $\mathrm{O}-\mathrm{H}$ $\left(\delta_{\mathrm{O}-\mathrm{H}}\right)$ at $891 \mathrm{~cm}^{-1}$. After liquiritin was dissolved in NADES, $v_{\mathrm{C}=0}$ shifted from $1,604 \mathrm{~cm}^{-1}$ to $1,610 \mathrm{~cm}^{-1}$, $v_{\mathrm{C}-\mathrm{O}-\mathrm{C}}$ shifted from $1,229 \mathrm{~cm}^{-1}$ to $1,231 \mathrm{~cm}^{-1}$, and $\delta_{\mathrm{O}-\mathrm{H}}$ shifted from $891 \mathrm{~cm}^{-1}$ to $893 \mathrm{~cm}^{-1}$. The spectral characteristics of liquiritin in NADES disclose multiple interactions between liquiritin and NADES.

\section{Discussion}

\section{Honey-frying enhances the immunostimulatory efficacy of licorice}

The pharmacological experiments confirmed that honey enhances the immunomodulatory efficacy of licorice by increasing macrophage phagocytosis and improving spleen and thymus function. Cell-based bioactivity experiments showed that the health-improving function of licorice was associated with its influences on the signal transduction pathways ER- $a$, TLR4, and Nrf2. The fortified immunomodulatory efficacy of licorice by honey-frying might be contributed to the improved content of total flavonoids and polysaccharides (Fig. 2). Polysaccharides enhance phagocytic activity in macrophages, promote immune organ development [26]. Metabolomics analysis (Fig. 3a) showed that honey-processing increased chalcone (isoliquiritin apioside (4), isoliquiritin (5), and O-acetylated derivatives $(14,16)$ ) levels which, in turn, increase the lymphocyte and macrophage abundance and CD4+/CD $8+$ cell ratios in the blood and regulate human immunity $[27,28]$. These results provide clear biological and chemical evidence for the relatively increased immunostimulatory efficacy of honey-processed licorice. These findings provide evidence for the traditional Chinese medicine theory about the processing of licorice, which says that honey-fried licorice "invigorates the spleen and qi (energy)".

\section{NADES characteristics of honey fortify the health-improving functions of honey-processed licorice.}

Our former studies proved that the major components of honey form a natural deep eutectic solvent composed of glucose, fructose, and sucrose and certain amount of water $[14,18]$. Hence honey does show the characteristic of NADES, including improving the dissolution and extraction of constituents, improving their thermal stability and bioavailability. The increased level of total flavonoids and polysaccharides in Fig. 2a prove that both honey and NADES enhance the dissolution and extraction of the active ingredients in the final licorice decoction, indicating that this effect of honey is due to its NADES characteristics. The total flavonoid content decreased in fried licorice and increased in 
honey/NADES-fried licorice (Fig. 2a) indicating honey/NADES may inhibit heat-induced decomposition of the flavonoids and at the same time increase the extraction of the flavonoids.

The protective effect of honey/NADES on the thermal stability of chemical constituents in licorice was verified in some single compounds in licorice (Fig. 3a and S1). Dihydroflavones ( 1 and 2 ) may have been isomerized to chalcone (compounds 4 and 5 ) due to the high temperature during processing (Fig. 3) [2, 5, 6], however, honey and NADES might suppress this transformation. Similar effect of honey /NADES on the stability of compounds was also observed. The observed decrease in glycosylated dihydroflavone level (1 and 2 ) after frying indicates that glucoside bonds may break during processing (Fig. 3) [2, 5, 6]. The relative levels of the isoliquiritigenin (6) differed from those of glycosylated chalcone $(4,5,14,16)$ among treatments. In contrast, the level of 2,4-dihydroxyacetophenone (9) was increased considerably by the frying, similar to the glycosylated chalcone (4) and to a less extend, the related compounds 5, 14, 16 (Fig. 3a and S1). Isoliquiritigenin (6) might degrade to 2,4-dihydroxyacetophenone (9) (Fig. 3b), whereas, honey/NADES may inhibit this heat-induced transformation. The major saponins in licorice is glycyrrhizic acid (7). The relative lower level of glycyrrhizic acid (7) and higher level of glycyrrhetinic acid (8) in $F$ than R (Fig. 3a) indicates that glycyrrhizic acid (7) may be degraded into glycyrrhetinic acid (8) during frying [2], whereas honey or NADES could inhibit this decomposition since higher level of glycyrrhizic acid (7) was observed in $\mathrm{H}$ and $\mathrm{N}$.

The effect of honey/NADES on the bioavailability of constituents in honey-fried licorice were demonstrated in Fig. 4 and Table S7. A pharmacokinetic analysis (Table S7) showed that there is no difference in the $\mathrm{AUC}_{0-12 / 24 \mathrm{~h}}$ values of four compounds between the $\mathrm{F}$ group and the $\mathrm{R}$ group, whereas the $A \mathrm{CC}_{0-12 / 24 \mathrm{~h}}$ values of liquiritin apioside (1), liquiritigenin (3) and glycyrrhetinic acid (8) were significantly higher when fried with honey or NADES than those of R. It indicates that honey improved the bioavailability of certain chemical constituents of licorice. NADES also increased the bioavailability of other compounds $[18,29,30]$. Overall, NADES in honey contributes to enhancing the health-improving functions of honey-fried licorice.

\section{Mechanism for the effect of honey on licorice}

The FT-IR measurements show that NADES and honey have similar chemical structures (Fig. 5). This finding confirms those of previous ${ }^{1} \mathrm{H}$ NMR and DSC analyses that NADES and honey had similar supramolecular structures $[15,18]$. The FT-IR measurements demonstrated that NADES interacts with liquiritin (2). This discovery is in agreement with reported molecular interactions including hydrogen bonds between quercetin and NADES [17]. Interactions between the solutes and NADES account for the ability of NADES and honey to stabilize and solubilize the bioactive constituents of licorice as well as improve their bioavailability, which result in enhanced biological activity of honey/NDES-fried licorice.

The improved bioactivities of honey-processed and NADES-processed licorice decoctions were confirmed, which is attributed to their NADES characteristics. Honey affects the solubility of the compounds in the plant material. As a rule, solubility of the aforementioned compounds is much higher in supramolecular liquids such as NADES than in water [14-18]. During decocting, NADES may liberate physically bound 
compounds from the plant matrix, interact with them, and increase their concentrations in the final decoction.

NADES may also stabilize licorice phenolics. The experiments herein indicated that chemical transformations occurred during processing. Figure $3 \mathrm{~b}$ showed that isomerization, glycolysis, and decomposition of chalcones to 2,4-dihydroxyacetophenone (9) might have occurred for fried licorice. The addition of honey inhibited chalcone isomerization and breakdown to 2,4-dihydroxyacetophenone caused by high temperature. However, it had no significant effect on glycosidic bond breakage. Previous studies $[2,5,6]$ reported that licorice flavonoids undergo isomerization and glycolysis during processing.

However, in general, phenolic compounds are relatively more stable in NADES than in water $[15,16]$. This study also provides bases for the effect of honey on quality improvement of natural products.

\section{Conclusion}

In conclusion, this study verifies that the ancient honey-frying processing of licorice enhances the immunomodulatory efficacy of licorice. The improvement is due to the NADES characteristics of honey, which inhibit heat-induced transformations of the bioactive compounds in licorice, improved their dissolution, and enhances their bioavailability after oral licorice decoction administration. These effects of honey are due to the mechanism that honey forms molecular interactions with compounds in licorice. These findings indicate that the biological activities of medicinal plants might be fortified by NADES in the processing of the plant material. The use of NADES for this purpose is not only improving the activity, it is also stabilizing the bioactive constituents present in the material. These findings provide theoretical and empirical bases for augmenting the beneficial health effects of herbal medicines using honey or other NADES. Further in depth studies may lead to novel potential industrial applications of honey or other NADES to fortify the health effects of herbal medicines.

\section{Abbreviations}

ANOVA, analysis of variance; AUC, area under the curve; BW, body weight; CE, collision energy; CS, calibration standard; DMEM, Dulbecco's modified Eagle's medium; DMSO, dimethyl sulfoxide; DP, declustering potential; DSC, differential scanning calorimetry; ESI, electrospray ionization; FBS, fetal bovine serum; FT-IR, Fourier transform infrared; HESI, heat electrospray ionization; UHPLC, ultrahighperformance liquid chromatography; ICR, Institute of Cancer Research; IS, internal standard; LPS, lipopolysaccharide; MRM; multiple reaction monitoring; MS, mass spectrometry; NADES, natural deep eutectic solvent; NMR, nuclear magnetic resonance; OD, optical density; OPLS-DA, orthogonal partial least square discriminant analysis; PBS, phosphate-buffered saline; PCA, principal component analysis; QC, quality control; RH, relative humidity; $\mathrm{SD}$, standard deviation; UHPLC, ultrahigh-performance liquid chromatography; TCM, Traditional Chinese medicine; VIP, variable importance in projection

\section{Declarations}




\section{Competing interests}

The authors declare no conflict of interest.

\section{Authors' contribution}

YD and RV conceived and designed the study, SK carried out the experiment and drafted the manuscript.

PL contributed analysis tools. JW contributed to the data analysis. YD, RV, CZ and SC revised the paper. All authors read and approved the final manuscript.

\section{Availability of data and materials}

All data included in this article are available from the corresponding author upon request.

\section{Acknowledgements}

The authors gratefully acknowledge Elsevier for its language proofreading service. The authors also thank the Center of Pharmaceutical Technology of Tsinghua University (Guan Wang and Weihua Wang, engineers) for the UHPLC-Q-Orbitrap MS analyses.

\section{Funding}

This work was supported by the National Natural Science Foundation of China [grant number 81803734] and the Project of China Academy of Chinese Medical Sciences [grant number ZXKT17009].

\section{Ethics approval and consent to participate}

All procedures were conducted in strict accordance with the Guide for the Care and Use of Laboratory Animals of the Ministry of Science and Technology of China (2006) and approved by the Animal Welfare Ethics Committee of the Institute of Chinese Materia Medica (China Academy of Chinese Medical Sciences, Beijing, China).

\section{Consent for publication}

All authors agree to publish this paper.

\section{References}


1. Sun L, Zhang T, Zhou Q, Shi D, Zhang L, Dai Y. Summary of the processing methods of licorice in the past. Chin Tradit Pat Med. 2010;32(07):1188-90.

2. Wang $M$, Zhang M, Tang Q, Li X. Influence of honey-roasting on the main pharmacological activities and the water-soluble active glycosides of licorice. Afr J Tradit Complement Altern Med. 2012;9(2):189-96.

3. Zhang H, Jia Y. Effects on immunological activities of Glycyrrizae Radix et Rhizome pieces before and after processed with honey. Journal of Pharmaceutical Research. 2018;37(08):446-8 + 459 .

4. China Pharmacopoeia Committee. Pharmacopoeia of People's Republic of China (Vol. 区), China Medical Science Press, Beijing, China, 2020.

5. Lu Y, Luo H, Wang L, Liu C, Zhang X, Yu X, et al. Analysis on Content Change and Transformation Rule of Main Characteristic Components in Stir-frying Process of Glycyrrhizae Radix et Rhizoma. Chin J Exp Tradit Med Formulae. 2020;26(10):1005-9903.

6. Ota M, Xu F, Li Y, Shang M, Makino T, Cai S. Comparison of chemical constituents among licorice, roasted licorice, and roasted licorice with honey. J Nat Med. 2018;72(1):80-95.

7. Alvarez-Suarez JM, Gasparrini M, Forbes-Hernández TY, Mazzoni L, Giampieri F. The Composition and Biological Activity of Honey: A Focus on Manuka Honey. Foods. 2014;3(3):420-32.

8. Escuredo 0 , Míguez M, Fernández-González M, Seijo MC. Nutritional value and antioxidant activity of honeys produced in a European Atlantic area. Food Chem. 2013;138(2-3):851-6.

9. Bogdanov S, Jurendic T, Sieber R, Gallmann P. Honey for nutrition and health: a review. J Am Coll Nutr. 2008;27(6):677-89.

10. Guo P, Deng Q, Lu Q. Anti-alcoholic effects of honeys from different floral origins and their correlation with honey chemical compositions. Food Chem. 2019;286:608-15.

11. Bobis O, Moise AR, Ballesteros I, Reyes ES, Durán SS, Sánchez-Sánchez J, et al. Eucalyptus honey: Quality parameters, chemical composition and health-promoting properties. Food Chem. 2020;325:126870.

12. Buba F, Gidado A, Shugaba A. Analysis of Biochemical Composition of Honey Samples from NorthEast Nigeria. Biochem Anal Biochem. 2012;2(3):139.

13. Brudzynski K, Miotto D, Kim L, Sjaarda C, Maldonado-Alvarez L, Fukś H. Active macromolecules of honey form colloidal particles essential for honey antibacterial activity and hydrogen peroxide production. Sci Rep. 2017;7(1):7637.

14. Choi Y, Spronsen J, Dai Y, Verberne M, Hollmann F, Arends I, et al. Are natural deep eutectic solvents the missing link in understanding cellular metabolism and physiology? Plant Physiol. 2011;156(4):1701-5.

15. Dai Y, Van Spronsen J, Witkamp GJ, Verpoorte R, Choi Y. Natural deep eutectic solvents as new potential media for green technology. Anal Chim Acta. 2013;766:61-8.

16. Dai Y, Witkamp GJ, Verpoorte R, Choi Y. Natural deep eutectic solvents as a new extraction media for phenolic metabolites in Carthamus tinctorius L. Anal Chem. 2013;85(13):6272-8. 
17. Dai Y, Verpoorte R, Choi Y. Natural deep eutectic solvents providing enhanced stability of natural colorants from safflower (Carthamus tinctorius). Food Chem. 2014;159:116-21.

18. Dai Y, Jin R, Verpoorte R, Lam W, Cheng Y, Xiao Y, et al. Natural deep eutectic characteristics of honey improve the bioactivity and safety of traditional medicines. J Ethnopharmacol. 2020;250:112460.

19. Simmler C, Anderson JR, Gauthier L, Lankin DC, Mcalpine JB, Chen SN, et al. Metabolite profiling and classification of DNA-Authenticated licorice botanicals. JNatProd. 2015;78(8):2007-22.

20. Chao J, Dai Y, Cheng H, Lam W, Cheng Y, Li K, et al. Improving the concentrations of the active components in the herbal tea ingredient, Uraria crinita: the effect of post-harvest ovendrying processing. Sci Rep. 2017;7:38763.

21. Chen $Y$, Wang J, Wan D. Determination of total flavonoids in three sedum crude drugs by uv-vis spectrophotometry. Pharmacognosy Magazine. 2010;6(24):259-63.

22. Chen L, Wang L, Wu H, Cheng J, Liu R, Chai Y, et al. Optimization of Extraction Technology and Alcohol Precipitation Process for Water-soluble Polysaccharides in Meretrix meretrix by Orthogonal Test. Chin J Exp Tradit Med Formulae. 2016;22(05):18-21.

23. Qiao X, Song W, Ji S, Wang Q, Guo D, Ye M. Separation and characterization of phenolic compounds and triterpenoid saponins in licorice (Glycyrrhiza uralensis) using mobile phasedependent reversedphasexreversed-phase comprehensive two-dimensional liquid chromatography coupled with mass spectrometry. J Chromatogr A. 2015;1402:36-45.

24. Zheng Y, Duan W, Yang Y, Xu Z, Li C, Cheng L, et al. Comparative study of water characteristic components of Glycyrrhiza uralensis from three geographical regions by chemical pattern recognition combined with muti-component qualitative and quantitative analysis. China $\mathrm{J}$ Chin Mater Med. 2019;44(12):2544-51.

25. Zhang Y, Wang M, Yang J, Li X. The Effects of the Honey-Roasting Process on the Pharmacokinetics of the Six Active Compounds of Licorice. Evid Based Complement Alternat Med. 2018:1-9.

26. Cao Y, Li K, Qin X, Jiao S, Du Y, Li S, et al. Quality evaluation of different areas of Astragali Radix based oncarbohydrate specific chromatograms and immune cell activities. Acta Pharmaceutica Sinica. 2019;54(07):1277-87.

27. Martínez G, Mijares MR, De-Sanctis JB. Effects of Flavonoids and Its Derivatives on Immune Cell Responses. Recent Pat Inflamm Allergy Drug Discov. 2019;13(2):84-104.

28. Gao X, Wang W, Wei S, Li W. Review of pharmacological effets of Glycyrrhiza Radix and its bioactive compounds. China J Chin Mater Med. 2009;34(21):2695-700.

29. Faggian M, Sut S, Perissutti B, Baldan V, Grabnar I, Dall'Acqua S. Natural Deep Eutectic Solvents (NADES) as a Tool for Bioavailability Improvement: Pharmacokinetics of Rutin Dissolved in Proline/Glycine after Oral Administration in Rats. Possible Application in Nutraceuticals Molecules. 2016;21(11):1531.

30. Sut S, Faggian M, Baldan V, Poloniato G, Castagliuolo I, Grabnar I, et al. Natural Deep Eutectic Solvents (NADES) to Enhance Berberine Absorption: An In Vivo Pharmacokinetic Study. Molecules. 2017;22(11):1921. 
Figures
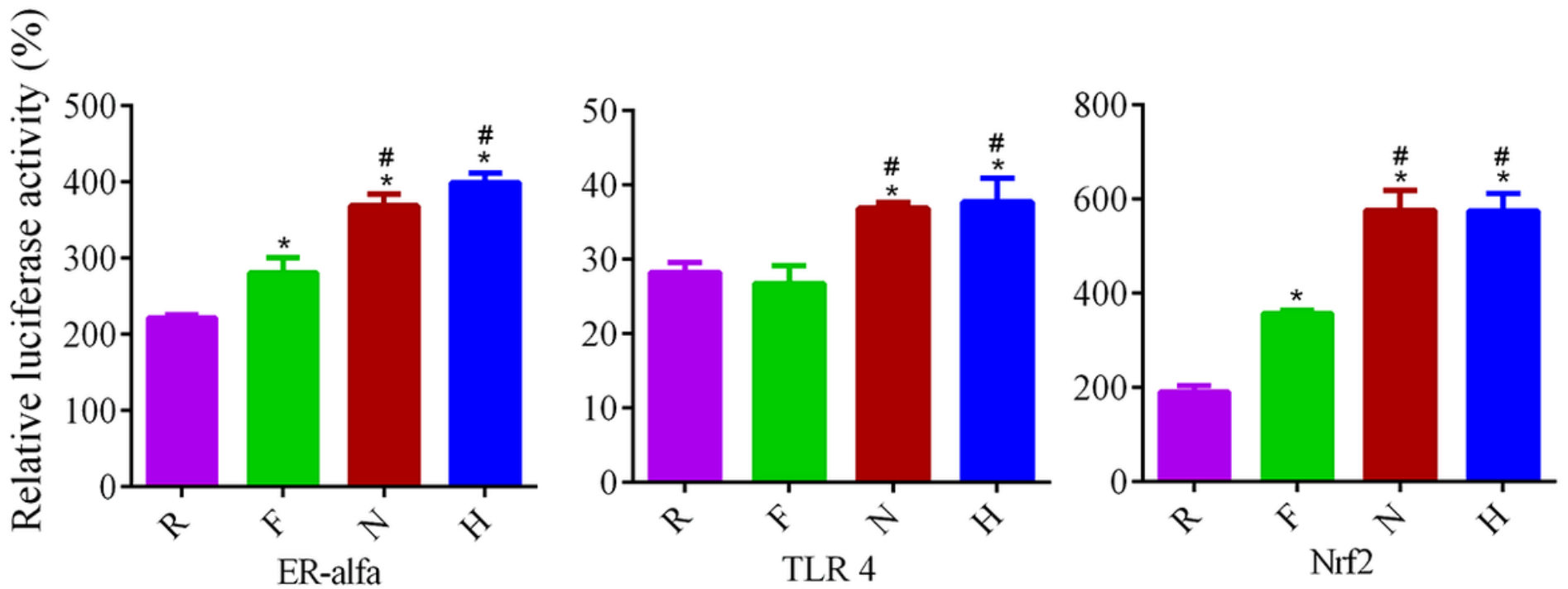

Figure 1

Cell-based bioactivities on endocrine-related ER-a, TLR4, and Nrf2 signaling pathways of different processed licorice decoctions. ( $R$, raw licorice; $F$, fried licorice; $H$, honey-fried licorice; N, NADES-fried licorice; $\mathrm{n}=6,{ }^{*} \mathrm{P}<0.05$ vs $\mathrm{R}$ group; $\# \mathrm{P}<0.05$ vs $\mathrm{F}$ group) 


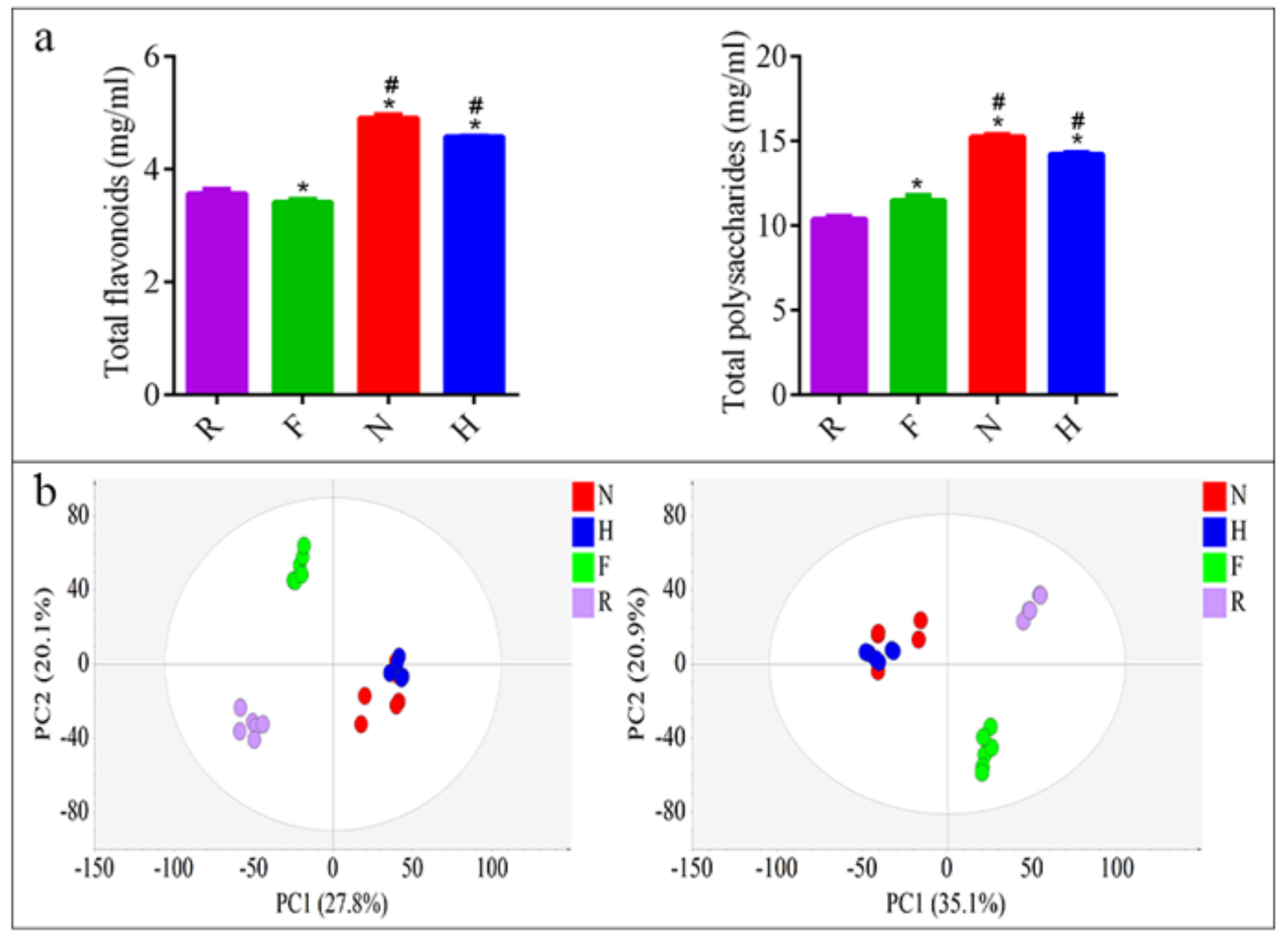

Figure 2

(a) The content of total polysaccharides and flavonoids of decoctions in different processed licorice. (b) PCA score plots for UHPLC-Q-Orbitrap MS based non-target metabolomics analysis in negative (left) and positive (right) mode. (R, raw licorice; $F$, fried licorice; $H$, honey-fried licorice; $N$, NADES-fried licorice; $n=6$, * $\mathrm{P}<0.05$ vs $\mathrm{R}$ group; $\# \mathrm{P}<0.05$ vs $\mathrm{F}$ group) 


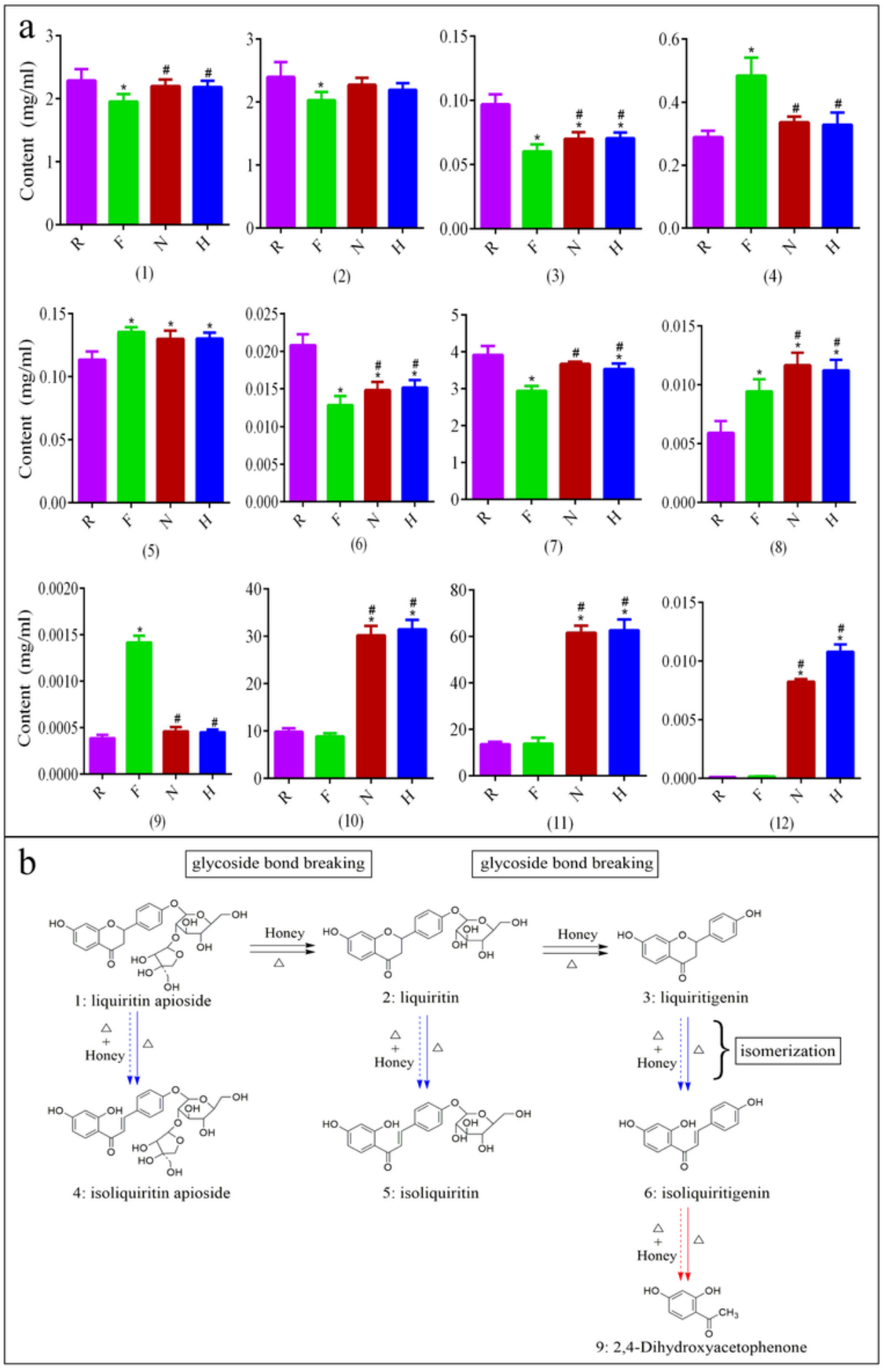

Figure 3

(a) Bar plots of the content of some quality difference markers. (b) Proposed conversion of chemical constituents in licorice caused by heat processing without and with honey or honey analogue (natural deep eutectic solvents, NADES). (R, raw licorice; $F$, fried licorice; $H$, honey-processed licorice; N, NADESfried licorice; $n=6$, * $P<0.05$ vs $R$ group; \# $P<0.05$ vs $F$ group. liquiritin apioside (1), liquiritin (2), liquiritigenin (3), isoliquiritin apioside (4), isoliquiritin (5), isoliquiritigenin (6), glycyrrhizic acid (7), 
glycyrrhetinic acid (8), 2,4-dihydroxyacetophenone (9), D-fructose (10), D-glucose (11), 5-

hydroxymethylfurfural acid (12); Solid line: conversion rate high; dotted line: relatively weak conversion rate; $\triangle$ : heat processing; $\triangle+$ Honey: heat processing with honey)
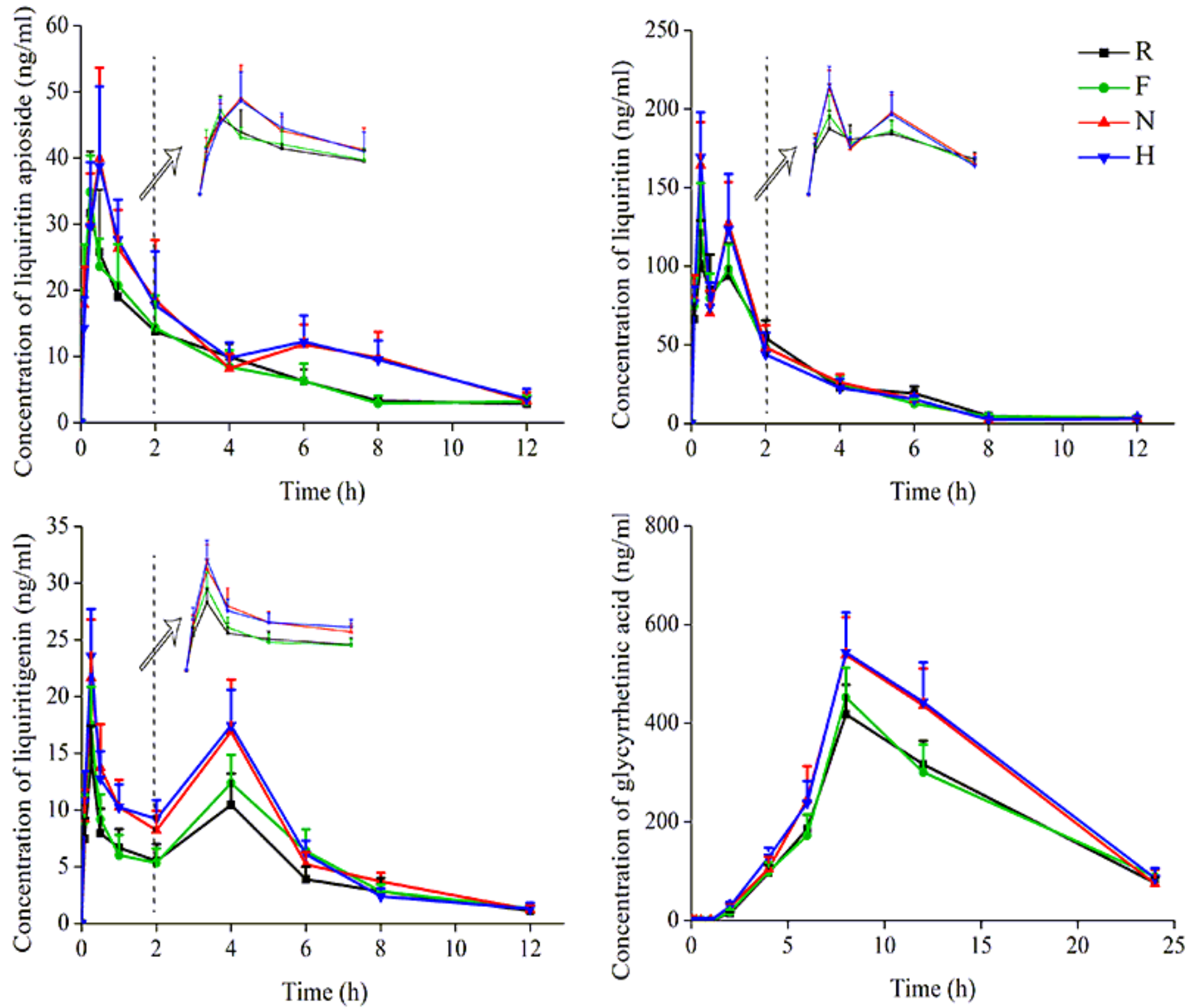

\section{Figure 4}

Plasma concentration-time profiles of liquiritin apioside (1), liquiritin (2), liquiritigenin (3), and glycyrrhetinic acid (8) after single oral administration of raw licorice decoction (8 g/ $\mathrm{kg}$ (raw licorice)), fried licorice decoction, honey-fried licorice decoction, honey analogue (natural deep eutectic solvents, NADES)-fried licorice decoction. 
a
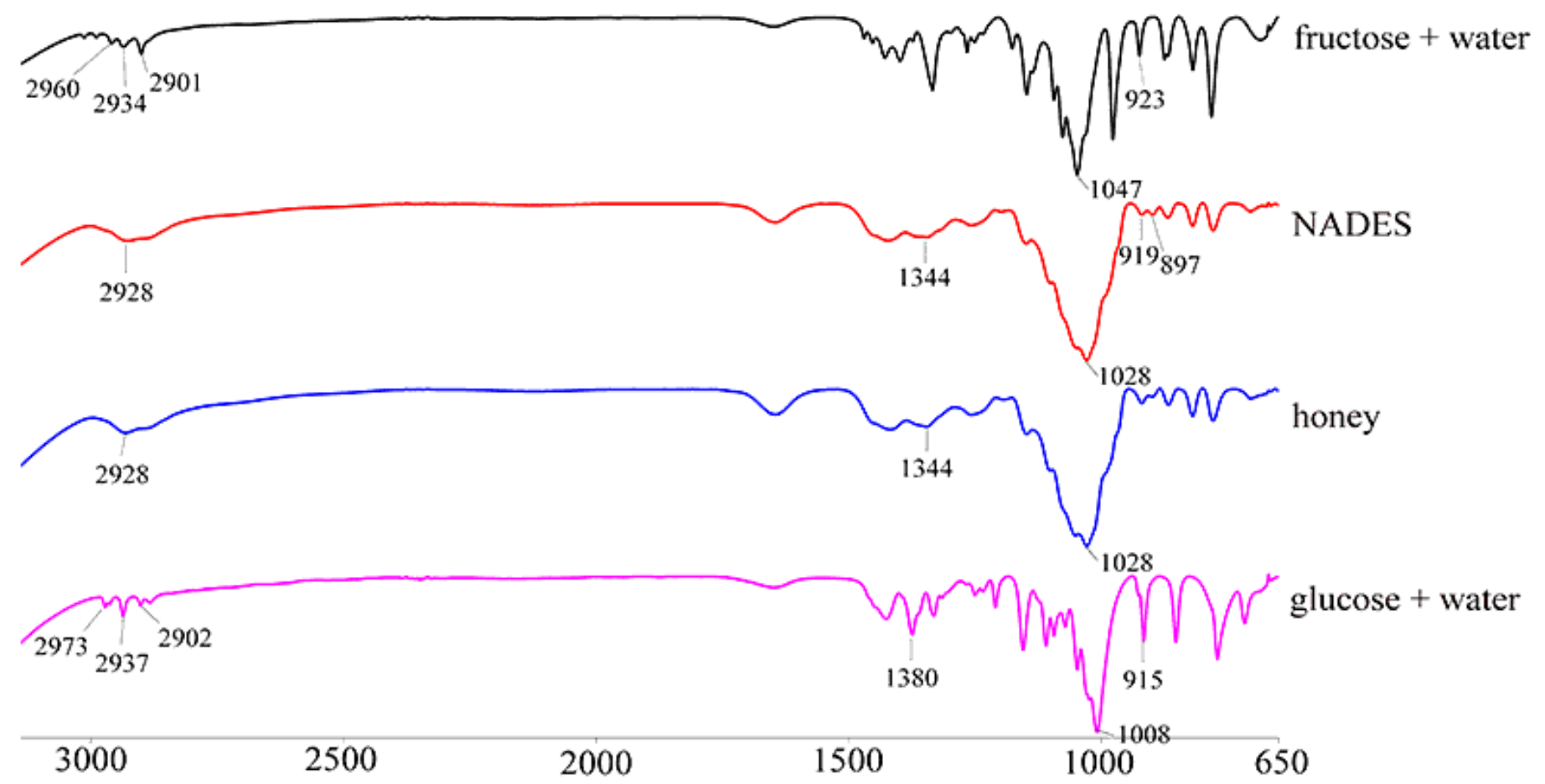

b

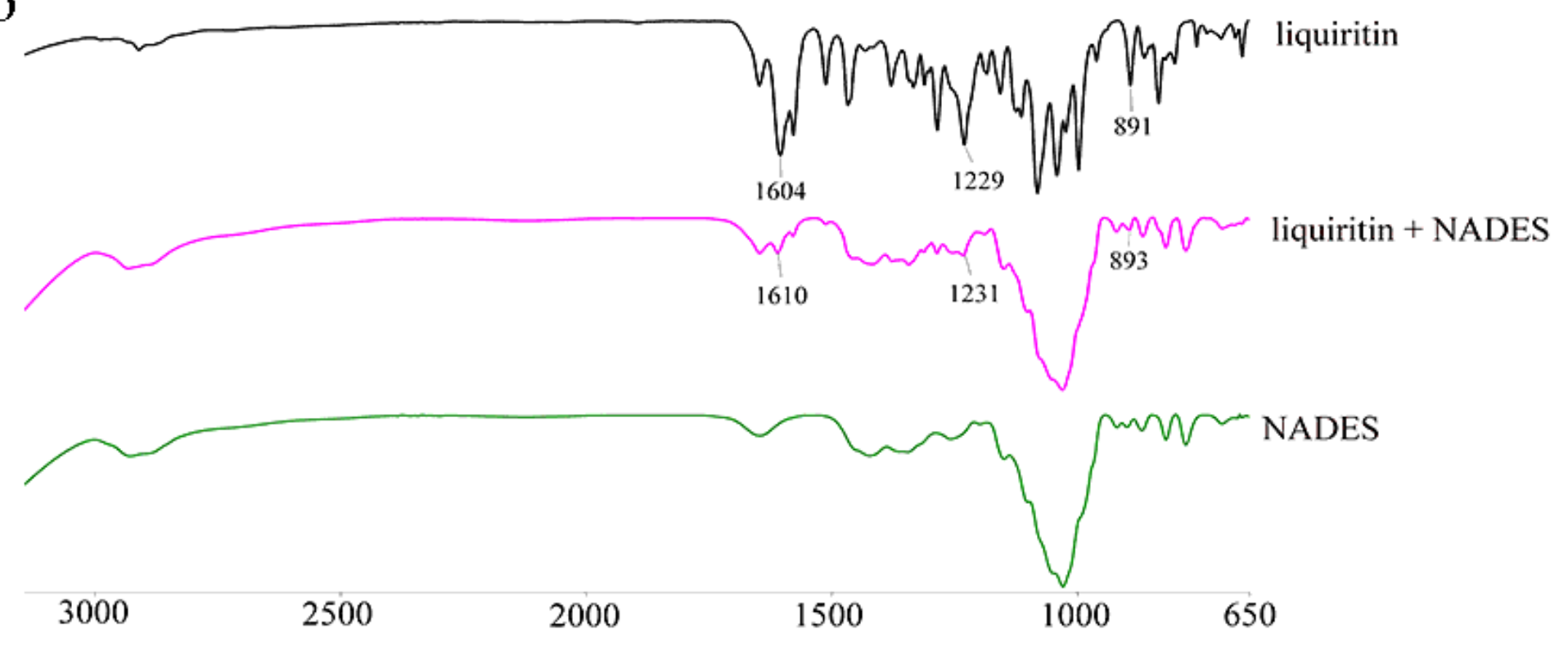

Figure 5

FT-IR spectra of (a) honey, honey analogue (natural deep eutectic solvents, NADES), fructose solution and glucose hydrate, and (b) liquiritin, a mixture of liquiritin and NADES, NADES.

\section{Supplementary Files}

This is a list of supplementary files associated with this preprint. Click to download. 
- SupplementaryMaterial.docx

- Figureabstract.png 